\title{
Dynamics of User Experience (UX)
}

\author{
Zahid Hasan \\ Shanto-Mariam University of \\ Creative Technology \\ Uttara, Dhaka, Bangladesh
}

\author{
Rathindra Chandra Gope \\ Shanto-Mariam University of \\ creative Technology \\ Uttara, Dhaka, Bangladesh
}

\begin{abstract}
User experience (UX) is not a static phenomenon; the way we interact with the technologies is dynamic which evolves over time. Ignoring this temporal nature of UX we cannot fully understand user experience. A longitudinal experiment was conducted over four weeks following 15 individuals using one of four medial players in their daily lives to examine users' behavior in prolonged use case and to investigate which factors have what type of impact on users' overall judgments at different point of time. Our analysis suggests that even if non-instrumental (e.g. Aesthetics of interface) quality plays an important role to form users overall judgments during the initial interaction, in prolonged use cage instrumental qualities (i.e. usability, functionality) become more influential.
\end{abstract}

\section{General Terms}

Human Computer Interaction

\section{Keywords}

User Experience, Usability, User satisfaction, User interface

\section{INTRODUCTION}

User Experience is a dynamic process triggered by diverse instrumental and non-instrumental aspects of products which evolve and change over time. Unfortunately most of the UX studies were conducted in a certain point of time rather than a range of period. This is why we are only able to see the glimpse of UX. Even the ISO definitions of UX and Usability do not emphasize the dynamic nature of user experience [1], and, with a few exceptions [10] [4] [5], this aspect has received little attention from the HCI community. We designed the evaluation method in such a way that it can give us an insight into users' behavior in prolonged use case. We evaluated both instrument and non-instrument quality of UX. After initial interaction with four media players, users were asked to choose one of them that they think the best according their choice and to use it for up to four weeks. They were farther asked to fill up the UX questionnaire during the second and fourth week. We collected data in four points in time (before interaction, after interaction, after two week and four week of daily use). In this way, we were able to investigate how users' behavior changes over time and which dimensions of UX had greater impact on different time frame.

\section{RELATED WORKS}

Wilamowitz-Moellendorff [10] described user experience (usage episode) as a series of single moments. Within these moments our experience may go through a usability problem, making us bored or some exciting features, giving us pleasure. At different phases of interaction we attach different weights to different product qualities. They suggest a timeline oriented interview technique (CORPUS: Change Oriented analysis of the Relationship between Product and User) as a viable alternative to longitudinal studies. This interview was designed to identify change in the perception of different quality dimensions during the last one to two years addressing pragmatic(utility, usability)and three hedonic dimensions (stimulation, beauty and identity) qualities of product. Their study revealed how mobile phones change their character over time: from the more hedonic to the more pragmatic: Pragmatic perceptions remained stable (utility) or even improved over time (usability) while hedonic perceptions (stimulation, beauty, communicate identity) deteriorated. Stimulation degrades because of frequent usage and beauty and identity were induced by comparisons with other people's products. There is evidence [4] that in our first interactions with a product we focus on its usability, aesthetics and the stimulation. After using it for some time, we might become less concerned about its usability, and other aspects of the product such as functionality or identity to others become more prominent. The longitudinal experiment can be divided into three groups: micro perspective (e.g., an hour), meso perspective (e.g., 5 weeks) and macro perspective, with a scope on years of use and the idea to map the whole product life cycle [10]. Most of the usability studies were done in micro level. We have limited number of study in meso level. Mendoza [8] observed over a period of eight weeks when users were creating websites. They found that the level of frustration decreased over time; the distribution of causes of frustration changed, and the users' responses to frustration episodes also changed. Their results suggest that those errors featured in conventional usability testing have little consequence over longer periods of time. In a comprehensive five week in-depth ethnographic' study, Karapanos et al. [5] examined the differences between initial and prolonged experiences in terms of the way users form overall evaluative judgments about products across time. They proposed dynamics of experience as consisting of three factors, i.e. familiarity, functional dependency and emotional attachment. These factors motivate the transition across the three phases of product adoption (i.e. orientation, incorporation and identification), and thus altering the way individuals experience a product over time. They define Anticipation as the act of anticipating an experience which happens prior to any actual experience of use. Orientation can be thought as the initial experience comprising feeling of excitement or frustration. As we continue to use the product, eventually we fall in the next phase of usage Incorporation where the product becomes meaningful in our daily lives and the product's usefulness becomes the dominant factor that constitute our overall evaluative judgments. The final phase is Identification, here we accept the product in our lives and it is supposed to participate in our social interactions, communicating our self-identity. Through the analysis they established the fact that perceptions of pragmatic quality were the primary determinant of the goodness of the product during early interactions, in prolonged experiences became the main determinant of goodness. However regarding to beauty judgments, their findings were contradictory to previous results (Hassenzahl, 2004 [3]) where beauty was found to be 
largely related to one's self-image (identification); instead their results showed stimulation to be even more prominent during the initial experiences. After four weeks of use, stimulation seemed to lose dominance on beauty judgments. Finally they concluded that product qualities that make initial experiences satisfying do not necessarily motivate prolonged use.

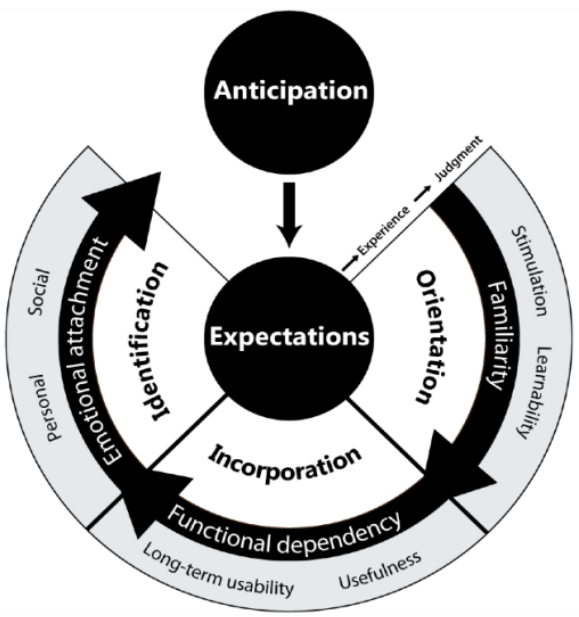

Fig 1: Experience across three phases: orientation, incorporation and identification proposed by [5]

\section{THE EXPERIMENT}

\subsection{Participants}

Fifteen Masters Students (14 M, 1 F; mean age $=26,4$ years) of a local University were involved, on a voluntary basis, as participants of the experiment. All the participants declared to have at least three years of experience in using media players.

\subsection{Settings and materials}

The materials used in the study were four freeware media players (iTunes, Music Bee, Media Monkey, and Songbird). The choice of the four media players was based on the fact that none of the participants had previous experience with any
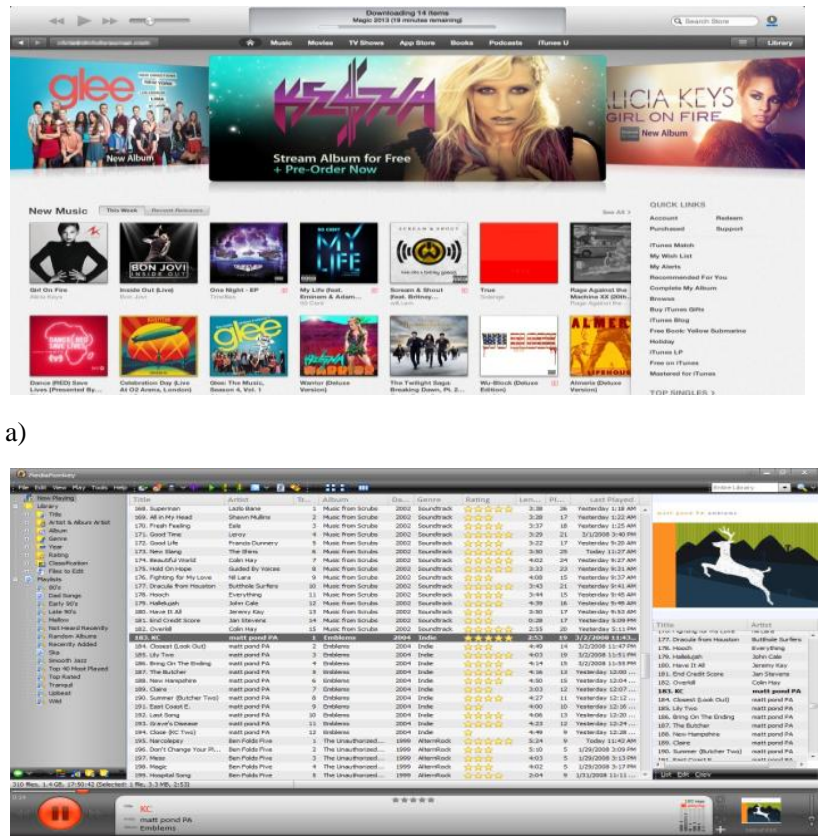

c) of them. The four media players used in the study had different look-and-feel, usability and functionality features; different sequences of actions were necessary to perform the tasks on each player. The tasks also differed in their level of complexity and in the minimum number of steps required to complete them (Table 1).

Task 1 (import a media folder) was relatively more difficult than the other two tasks for all three media players. However, iTunes and Songbird provided clear instructions on how to do it, while the procedure was less intuitive for Media Monkey and Music Bee. For example, iTunes and Songbird featured the words 'add folder' and 'Import' in the menu, whereas Media Monkey and Music Bee used the word 'Add/Rescan' which might be confusing for the user. iTunes and Songbird supported also drag-n-drop for importing media folder.

The sequence of actions required for task 2 (find and play a song) does not present significant differences among the media players. In all of them, songs can be sorted according to title, album, artist, genre and date; and all players also featured an internal search engine. However, they differed in terms of visual representation of the songs, with iTunes featuring the most appealing interface.

Task 3 (locating and applying the equalizer) was easier in iTunes, Songbird and Media Monkey than in Music Bee. This difference was due to the fact that in the first three mediaplayers the equalizer was located under one menu ('view', 'control' or 'tool') from where it could be directly assessed. In the case of Music Bee, the item equalizer could be found under 'view' and 'controls' menu. Selecting it from the controls menu does not produce any visible reaction on the system. Selecting Equalizer from the 'view' menu requires to select the 'player control panel' item and then the 'show equalizer' one. At this point the equalizer windows opens, and requires clicking a radio button to enable it. Both Music Bee and Media Monkey have an icon on the main interface for accessing the equalizer quickly, but these icons are difficult to understand and notice.

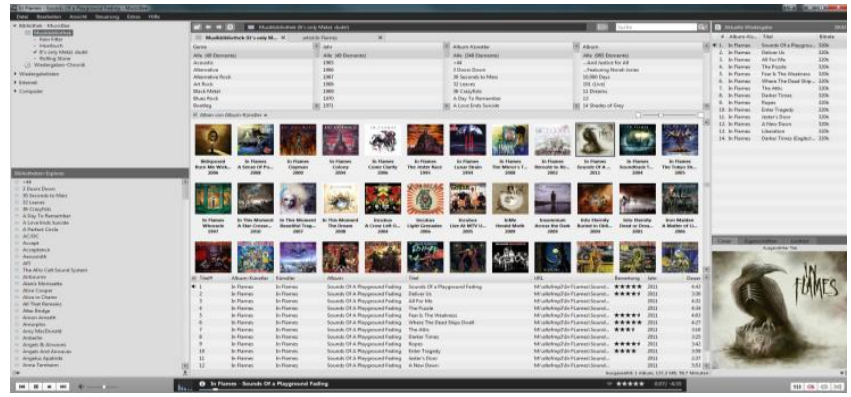

b)

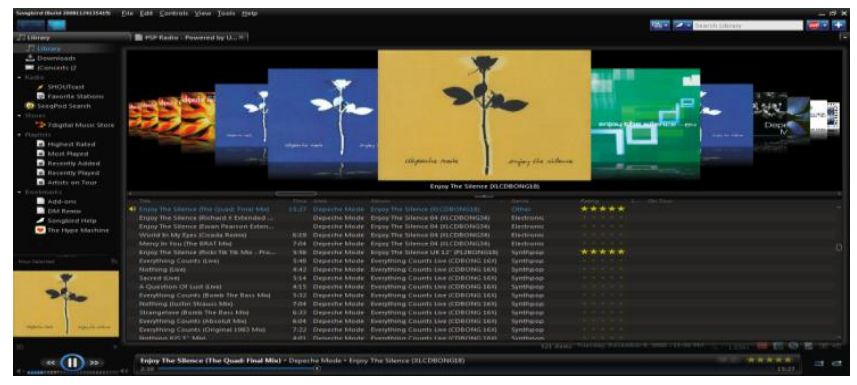

d)

Fig 2: The four media players: iTunes (a), Music Bee (b), Media Monkey (c) and Songbird (d). 
Table 1. The steps required to complete the tasks on the media players

\begin{tabular}{|c|c|c|c|}
\hline Media Players & Tasks & Strategies & Steps \\
\hline \multirow{5}{*}{ iTunes } & \multirow{2}{*}{$\begin{array}{l}\text { 1.Import } \\
\text { media folder }\end{array}$} & 1.Drag-and- drop & 1.Select folder 2.Move folder 3.Release folder on music panel \\
\hline & & 2. Menu based & $\begin{array}{l}\text { 1.Select 'File' menu 2.Select 'Add folder to library' item 3.Locate and select } \\
\text { media folder 4. Click OK }\end{array}$ \\
\hline & \multirow{2}{*}{$\begin{array}{l}\text { 2.Find and } \\
\text { play song }\end{array}$} & 1.Search engine & 1. Enter song title in search box 2. Click ok / press enter 3. Locate song 4. Play it \\
\hline & & 2.Browse in music panel & 1. Find the song in the music library 2. Play it \\
\hline & \multicolumn{2}{|l|}{$\begin{array}{l}\text { 3.Apply } \\
\text { equalizer }\end{array}$} & 1. Select 'View' menu 2.Select 'Show Equalizer' 3. Close it. \\
\hline \multirow{6}{*}{ Songbird } & \multirow{3}{*}{$\begin{array}{l}\text { 1.Import } \\
\text { media folder }\end{array}$} & 1.Drag-and- drop & \\
\hline & & & \\
\hline & & 2.Menu based & $\begin{array}{l}\text { 1.Select 'File' menu 2.Select 'Import media' item 3.Locate and select media } \\
\text { folder 4.Click OK }\end{array}$ \\
\hline & \multirow{2}{*}{$\begin{array}{l}\text { 2. Find and } \\
\text { play song }\end{array}$} & 1.Search engine & 1.Enter song title in search box 2.Click ok / press enter 3.Locate song 4.Play it \\
\hline & & 2.Browse in music panel & 1.Find the song in the music library 2.Play it \\
\hline & $\begin{array}{l}\text { 3. Apply } \\
\text { equalizer }\end{array}$ & 1.Menu based & 1.Select 'Control' menu 2.Select 'Equalizer' item 3.Close it \\
\hline \multirow{6}{*}{ MediaMonkey } & \multirow{2}{*}{$\begin{array}{l}\text { 1.Import } \\
\text { media folder }\end{array}$} & 1.Menu based & $\begin{array}{l}\text { 1.Select 'File' menu } 2 \text { Select 'Add/Rescan tracks to library' item 3.Expand } \\
\text { folder Tree 4.Locate and select media folder 5.Clik OK }\end{array}$ \\
\hline & & $\begin{array}{l}\text { 2. Locate the media } \\
\text { folder form left menu }\end{array}$ & 1.Select Library 2.Select Location 3.Select media folder \\
\hline & \multirow{2}{*}{$\begin{array}{l}\text { 2. Find and } \\
\text { play song }\end{array}$} & 1.Search engine & 1.Enter song title in search box 2.Click ok / press enter 3.Locate song 4.Play it \\
\hline & & $\begin{array}{l}\text { 2.Browse in the music } \\
\text { panel }\end{array}$ & 1.Find the song in the music library 2.Play it \\
\hline & \multirow{2}{*}{$\begin{array}{l}\text { 3. Apply } \\
\text { equalizer }\end{array}$} & 1.Menu based & 1.Select 'View' menu 2.Select 'Equalizer' item 3.Close it \\
\hline & & 2.Icon based & 1.Select icon on the interface 2.Close it \\
\hline \multirow{7}{*}{ MusicBee } & \multirow{2}{*}{$\begin{array}{l}\text { 1.Import } \\
\text { media folder }\end{array}$} & 1.Menu based & $\begin{array}{l}\text { 1. Select 'File' menu 2. Select 'Add/Rescan File Insert' item 3. Expand folder } \\
\text { Tree 4. Locate and select media folder 5. Click OK }\end{array}$ \\
\hline & & $\begin{array}{l}\text { 2.Locate the media } \\
\text { folder form left menu }\end{array}$ & 1.Select 'Computer' item 2.Expand folder tree 3.Find and locate media folder \\
\hline & \multirow{2}{*}{$\begin{array}{l}\text { 2. Find and } \\
\text { play song }\end{array}$} & 1.Serach engine & 1.Enter song title in search box 2.Click ok / press enter 3.Locate song 4.Play it \\
\hline & & $\begin{array}{l}\text { 2.Browse in the music } \\
\text { panel }\end{array}$ & 1.Find the song in the music library 2.Play it \\
\hline & \multirow{3}{*}{$\begin{array}{l}\text { 3. Apply } \\
\text { equalizer }\end{array}$} & 1.a. Menu based & $\begin{array}{l}\text { 1.Select 'View' menu 2.Select 'Player Control Panels' item 3.Select 'Show } \\
\text { Equalizer' item 4.Select 'Controls' menu 5.Select 'Equalizer' item 6.Close it }\end{array}$ \\
\hline & & 1.b. Menu based & $\begin{array}{l}\text { 1. Select 'View' menu 2.Select 'Player Control Panels' item 3.Select 'Show } \\
\text { Equalizer' item 4. Mark 'select equalizer' checkbox 5.Close it }\end{array}$ \\
\hline & & 2. Icon based & 1.Select icon on the interface 2.Mark 'select equalizer' checkbox 3.Close it \\
\hline
\end{tabular}




\subsection{Procedures}

The experiment started with the experimenter showing to participants the screen shots of the four media players presented in random order for 10 seconds, with the purpose of collecting a first-impression evaluation of the interface, after each screen shot a set of questionnaires were filled up by them. During the second step of the study, participants were asked to perform the same three tasks on the four media players and to fill in the UX questionnaire immediately after completion of the tasks on one media player. The order in which participants used the four media players was randomized, while the order of the three tasks was the same for all the participants. The three tasks were:

1. Importing a folder containing songs to the media library of the player;

2. Finding a particular song in the media library and playing it;

3. Adjusting the equalization of the song by using the equalizer inbuilt in each media player.

Once participants completed the fourth questionnaire, they were asked to choose one of the 4 media-players and to commit to use it for the following month instead of their usual program.

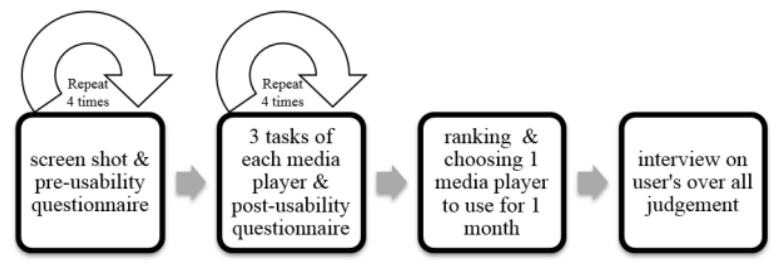

Fig 3: The flow the experiment

\subsection{Dependent variables}

The experiment included the following dependent variables: 1) Performance measures associated to task execution; 2) judgments about user experience provided by participants through questionnaires.

Performance measures. Errors were calculated by subtracting the number of steps performed by the participant and the minimum number of steps needed to complete the task. Task completion time was recorded in seconds.

User experience evaluation. The questionnaire was composed of 3 parts addressing the evaluation of media-players, information about participants' previous usage of mediaplayers, and basic demographic data. Media-players were evaluated for individual dimensions of UX (usability, aesthetics, symbolism, pleasure and functionality) and summary judgments.

4. Usability was measured by means of 4 items: Easy to use, Easy navigation, convenient use, Easy orientation [6].

5. A two-factor model of aesthetics was used which differentiates between classical and expressive aesthetics [6]. Classical aesthetics refers to traditional notions of beauty emphasizing symmetry, order and clear design. It includes 5 items: Clear design, Symmetric design, Clean design, Pleasant, Aesthetic design. Expressive aesthetics is characterized by qualities that capture the user's perception of the creativity and originality of the design: original, sophisticated design, creative design, use of special effects, fascinating.

6. Symbolism concerns the inference of connotative meanings associated to an interactive device [9]. As opposed to aesthetics, symbolism appraisal depends on cognitive processes. It included the following items: fits personality, creates positive associations, represents likable things, communicates desirable image, and provides a positive message about user. d. Pleasure was assessed by 3 items proposed in [6]: feel joyful, feel pleasure, and feel gratified.

7. The evaluation of functionality included 4 items: performs the tasks required, produces expected results, can interact with other software, can provide security. f. User satisfaction was based on 5 items from [7] and [2]: I will use it in the future, I would recommend it to friend, it will be fun to use, I feel I will need to have it, and I will be satisfied with it. Given the small size of the sample, no psychometric validation of the scales was possible. Dependent variables were computed by averaging relevant items for each UX dimension, as highlighted by a large corpus of previous research in [6] [2].

\section{RESULTS}

\subsection{Judgments about UX (In Use)}

Among fifteen users, nine $(60 \%)$ of them used VLC media players and the rest (six) of them (40\%) of them used WMP (Windows Media Player). In terms of aesthetics (both expressive classic dimensions), Windows media player (WMP) received better rating than VLC media player. However, VLC outperformed WMP in all other dimensions including usability, symbolism, pleasure, functionality and overall judgment rating (main effect of media players on expressive aesthetics and usability are $(\mathrm{F}(1,13)=7.488, \mathrm{p}$ $<.05)$ and $(F(1,13)=6.294, p<.05)$ respectively. It is worth to notice that for both of these media players usability got the highest rating than the other UX dimensions.

A One Way ANOVA analysis revealed that the main effect of averaged UX dimensions is significant $(F(6,104)=4.616, p$ $<.001)$ and post hoc comparison indicated that this was due to high scores of usability followed by pleasure and satisfaction. This is due to the fact that users have been using this media player for long time. This result conforms to the findings of [5] and [10] where in prolonged use case (once users incorporate the product into their life) usability dominates to form the overall evaluative judgments about products.



Fig 4: UX Ratings of Media Players in use. 


\subsection{Judgments about first expression}

Before interacting with the media players users were briefly (10s) exposed to the screen-shot of these four media players. After each screen-shot they were requested to fill up the UX questionarraire. The purpose of this step was to investigate the impact of first impression on judgment about perceived usability and performance (actual usability). Pre-use aesthetic and perceived usability judgments were analyzed followed by post-use aesthetic and perceived usability judgments. We also analyzed whether there is any relationship between aesthetics and actual usability (i.e. error $\&$ time).

Strong correlation was found between perceived usability and classic aesthetics $(\mathrm{r}=.569)$ but not with expressive aesthetics $(\mathrm{r}=.022)$ prior to actual interaction with the media players. Since classic aesthetics emphasizes on orderly and clear design which is closely related to usability, whereas expressive aesthetics is related to the designers' creativity, originality and the ability to break design, it can be inferred that classical aesthetics should be more correlated with usability factors. However after the interaction both expressive and classic aesthetics correlated moderately with post usability ( $r=.472$ for expressive $\& \mathrm{r}=.422$ for classic).

Those who registered a high score on the classic aesthetics measure also registered a high score on the perceived usability measure before the actual task. These correlations resemble to the findings by Tractinsky et al [11]. No correlation was found for pre aesthetics Judgments and post-use errors made ( $r=-0.044$ for classic \& $r=-0.037$ for expressive) or completion times $(r=0.089$ for classic \& $r=0.036$ for expressive). Therefore, aesthetic perceptions had no relation to performance. These findings contrast with the claims of Norman [12]; who argued that positive emotion causes people to be more creative in thinking and brainstorming which lead to overcome the difficulties. Thus we may conclude that if a user perceived the look-n-feel of the software to be beautiful then he also believed it to be usable even after the use of the media players.

\subsection{Judgments about UX (Pre Use)}

Performance Analysis A sample of 168 tasks was collected and included in the following analyses, where task was used as the unit of analysis. The average number of error per task was 3.98 ( $\operatorname{std} \mathrm{dev}=7.14$ ), ranging from a minimum of 0 to a maximum of 39. The distribution shape of errors was improved by computing the square root of each data-point. This normalized variable was analyzed by an ANOVA with media-player (4) and task (3) as the between-subjects factor. Post-hoc comparisons were based on the Least-Significance Difference method. The main effect of media-player $(\mathrm{F}(3,156)$ $=6.73, \mathrm{p}<.001$, partial $2=.12)$ and task were significant $(\mathrm{F}(2,156)=7.83, \mathrm{p}<.01$, partial $2=.09)$. The Post-hoc analysis suggested that the media player effect was due to the poor performance of Musicbee whereas no differences emerged between the other media-players. The effect of task was due to task 2 which was significantly easier than the other two tasks.

The results of the Anova on the square root of time highlighted a very similar pattern. The main effects of media player $(\mathrm{F}(3,156)=5.85, \mathrm{p}=.001$, partial eta squired $=.10)$ and task were significant $(\mathrm{F}(2,156)=7.70, \mathrm{p}<.001$, partial eta squired $=.09$ ). Post-hoc analysis highlighted that Musicbee took the longest time to perform the tasks (with no significant differences between the other media players), and task 2 was significantly faster than the other tasks. A high linear correlation between error rate and execution time was found $(\mathrm{r}(168)=.88, \mathrm{p}<.001)$

Subjective evaluations collected by questionnaires at the end of the third task showed little correlation with error numbers. The values for functionality $(r=.20)$ pleasure $(r=-.15)$ and overall judgment $(\mathrm{r}=-17)$ were significant. Interestingly, all these correlations were due to the performance of participants at task 3. Task 1 and task 2 returned no correlations at all, whereas task 3 reported higher and significant correlations for all variables but expressive aesthetics.

In case of average errors, clearly Musicbee performed the poorest; it also took the highest time to perform. On the other hand, iTunes, Songbird and Media-monkey were close to each other in terms of both errors and time.

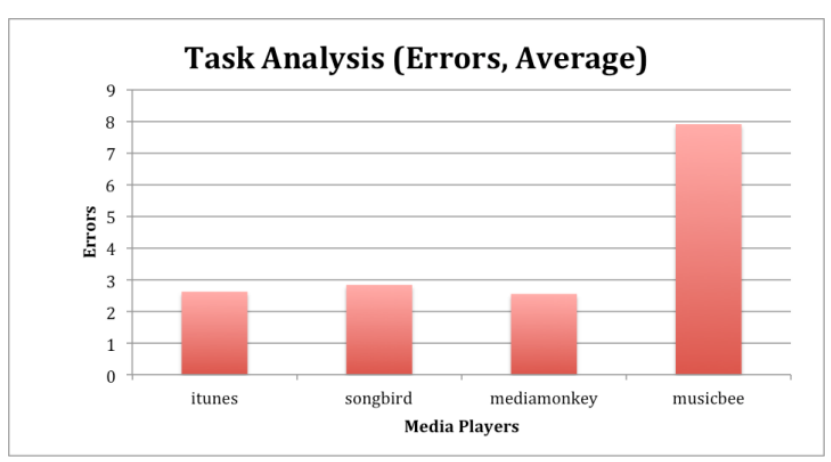

Fig 5: Task Analysis (Error)

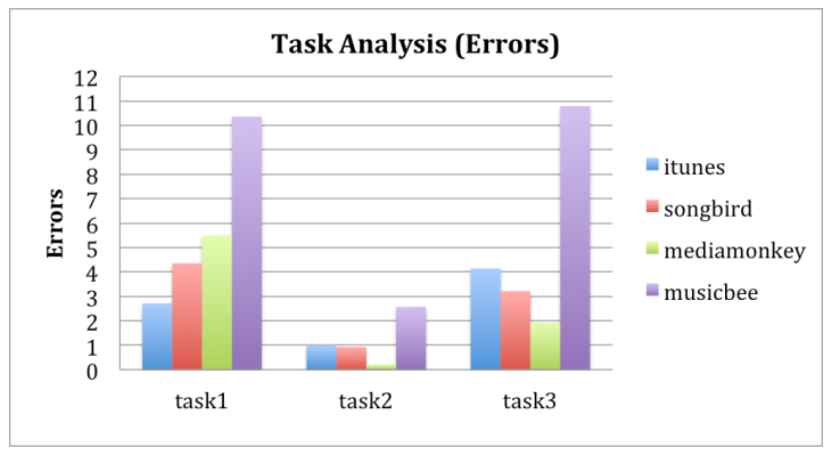

Fig 6. Task Analysis (average Error)

\subsection{Judgments about UX (Post Use)}

In all UX dimensions, I-tunes were perceived and evaluated as the best system (average rating well above 5). There are not much differences between Songbird and Media-monkey (average rating between 4 and 5), while Musicbee was scored more negatively, with significant differences between usability, functionality, pleasure and overall judgment (average rating below 4). These trends of results are consistent with participants' final choice of the media-player to use for the next month. Indeed, 10 people decided to use i-tunes, 3 people choose Songbird, Media Monkey was chosen only by 2 people and nobody decided to use Musicbee.

Although there were no noticeable differences in case of errors made among iTunes, Songbird and Musicbee, nevertheless, iTunes received the highest rating in all UX dimensions; it appeared to be more beautiful, usable and functional to the users. We see the reflection of this fact when people rated iTunes as the most desired media players and $66.66 \%$ of the users decided to use it. 




Fig 7: UX Ratings of Media Players (post use)

\subsection{Longitudinal assessments}

10 out of 15 users decided to use iTunes for up to four weeks and we received UX data at four points of time (before interaction, after interaction, after two week and four week of daily use), the figure below (Fig.8) shows the general trend of the six UX dimensions (expressive \& classic aesthetics, usability, symbolism, pleasure and functionality) over time for iTunes. We took only iTunes into account because the other two media players, Songbird and Media monkey, were chosen by only 3 and 2 users respectively. It is interesting to notice that there is a sharp fall for functionality and overall judgments from second to third phase (fig. 8); however, they remain nearly constant from third to fourth phase. A one way repeated measure ANOVA with time (4 phases) as an independent variable and seven UX dimensions as dependent variables was performed. There was a significant main effect of time on UX dimensions (Wilks Lambda $=0.1, \mathrm{~F}$ (21, $60.685)=2.242, p=.008$, partial eta squared $=0.422$ ). This effect was high for functionality $(\mathrm{F}(3,27)=12.049, \mathrm{p}<.001$, partial eta squared $=0.572)$ and satisfaction $(\mathrm{F}(3,27)=$ $16.357, \mathrm{p}<.001$, partial eta squared $=0.645$ ). Pairwise comparisons suggest that these changes occur between phase 2 and phase 3 . On the other hand judgment of pleasure ( $F(3$, $27)=9.332, \mathrm{p}<.001$, partial eta squared $=0.509)$ and symbolism $(\mathrm{F}(3,27)=4.712, \mathrm{p}<.009$, partial eta squared $=0.344$ ) decline moderately through all of four phases, whereas there is no noticeable changes on usability and beauty judgments for both classic and expressive dimensions.

\section{DISCUSSION}

These changes can be explained by the model proposed by [5]. According to this model, from phase 1 to phase 2 can be viewed as orientation; from phase 2 to phase 3 is similar to incorporation and from phase 3 to phase 4 can be regarded as identification in their model. They suggested that in corporation phase product becomes meaningful in our daily lives. In this phage long-term usability as well as usefulness becomes the major factor impacting our overall evaluative judgments. The underneath reason behind the dramatic fall of overall judgment of iTunes is due to the fact that none of the users incorporated iTunes into their daily life; in fact, they found the lack of functionality in iTunes as it cannot play video files, whereas the media players they used had the capability of playing both audio and video files. Moreover, several users reported that iTunes tends to consume more computer resources and bit slower comparing VLC media player that they used before the experiment. Since iTunes failed to be incorporated into their lives, we cannot see much variation in Identification phase, only symbolism and pleasure which are the key determinant of this phase continue to decline.



Fig 8: iTunes over four point of time.

\section{CONCLUSION}

User experience can be divided into a series of single moments, according to [4], which is 'usage episode'. Within these moments our experience may go through a usability problem, making us bored or some exciting features, giving us pleasure and we momentarily form an overall judgment (i.e. Hasenzahls goodness beauty) about the product. Each different phase of UX is weighted by different product qualities. As the experiences go through, the new usage episode is formed not only by the current product qualities but also the experience of the previous usage episode. The temporality of UX can be distinguished into three phases: preinteraction phase including anticipation/expectation, interaction phase where the actual usage with the system occurs, this phase consists of orientation, incorporation and 
identification and finally post-interaction phase where users no longer use the product but carry the emotional experience that they had. This work suggests that at the very early stage of interaction users' overall judgments are formed mainly by aesthetics of the interface and perceived usability (perceived usability is also influenced by aesthetics). When the actual interaction begins, both pragmatic (usability, functionality) and hedonic qualities (symbolism, stimulation, and aesthetics) contribute to overall judgments. In the orientation phase usability is the main determinant whereas in the incorporation phase functionality comes into play along with pleasure.

\section{REFERENCES}

[1] Bevan, N. What is the difference between the purpose of usability and user experience evaluation methods. In Proc. of the UXEM Workshop at INTERACT (2009).

[2] De Angeli, A., Sutcliffe, A., and Hartmann, J. Interaction, usability and aesthetics: what influences users' preferences? In Proceedings of the $6^{\text {th }}$ conference on Designing Interactive systems, ACM (2006), 271280.

[3] Hassenzahl, M. The interplay of beauty, goodness, and usability in interactive products. Human-Computer Interaction 19, 4 (2004), 319-349.

[4] Karapanos, E., Hassenzahl, M., and Martens, J. User experience over time. In CHI'08 extended abstracts on Human factors in computing systems, ACM (2008), 3561-3566.

[5] Karapanos, E., Zimmerman, J., Forlizzi, J., and Martens, J. User experience over time: an initial framework. In
Proceedings of the 27th international conference on Human factors in computing systems, ACM (2009), 729 738.

[6] Lavie, T., and Tractinsky, N. Assessing dimensions of perceived visual aesthetics of web sites* 1 . International Journal of Human-Computer Studies 60, 3 (2004), 269298.

[7] Lewis, J. Ibm computer usability satisfaction questionnaires: psychometric evaluation and instructions for use. International Journal of Human-Computer Interaction 7, 1 (1995), 57-78.

[8] Mendoza, V., and Novick, D. Usability over time. Proceedings of the 23rd annual international conference on Design of communication for pervasive information (2005), 151--158

[9] Tractinsky, N., and Zmiri, D. Exploring attributes of skins as potential antecedents of emotion in hci. Aesthetic computing (2006), 405-422.

[10] Von Wilamowitz-Moellendorff, M., Hassenzahl, M., and Platz, A. Dynamics of user experience: How the perceived quality of mobile phones changes over time.

[11] Tractinsky, N., Katz, A. S., Ikar, D. What is beautiful is usable. Interacting with Computers, (2000),13, pp.127145 .

[12] Norman, D. A. Emotion and design: Attractive things work better. Interactions Magazine, (2002) 9 (4), 36-42. 